\title{
NOUVELLE
}

\section{L'oligarchie contestée des cellules souches cancéreuses}

Sophia Häfner, Laure Coulombel
S. Häfner : Médecine/Sciences

et École Normale Supérieure, Paris, France.

Sophia.Hafner@inserm.f

L. Coulombel : Médecine/Sciences et Inserm U935, Hôpital Paul Brousse, 94817 Villejuif Cedex, France.

Icoulombel@medecinesciences.org

\section{Le dogme}

des cellules souches cancéreuses

Depuis quelques années, le concept de cellule souche cancéreuse (CSC) - ou de cellule capable de former une tumeur pour cancer initiating cell- a quasiment été élevé au rang de dogme selon lequel dans les tumeurs, par analogie avec les tissus normaux, seule une fraction très minoritaire de cellules (0,0001-0,1\%), qui peuvent être distinguées de la masse des cellules cancéreuses «différenciées » et à courte durée de vie, possède la capacité de s'auto-renouveler et de reconstituer l'hétérogénéité de la tumeur [1]. L'impact thérapeutique n'est pas mince, car contrairement à la majorité des cellules tumorales, les CSC pourraient être résistantes à la chimiothérapie et/ ou à la radiothérapie, expliquant ainsi certaines rechutes et s'opposant à l'éradication définitive de la maladie. Mais a t-on toujours une preuve expérimentale infaillible de la réalité de ces CSC, c'està-dire a t-on démontré à l'échelon unicellulaire que ces CSC reconstituaient la tumeur in vivo? Pas sûr, mais pas facile! Les arguments initiaux en faveur de la réalité de CSC proviennent de l'analyse de certaines leucémies myéloïdes [2, 3], où une petite sous-population $(<0,001 \%)$ de cellules, de phénotype $\operatorname{CD} 34^{+} / \operatorname{CD}^{-} 8^{-}$(un phénotype que partagent aussi les CS hématopoïétiques normales), est seule capable de générer les populations postmitotiques cancéreuses après injection à des souris immunodéficientes NOD-SCID, alors que le reste des cellules leucémiques en est incapable; des arguments convaincants ont aussi été obtenus dans quelques tumeurs solides, notamment les glioblastomes et dans ce cas, les CSC se distinguent par l'expression de l'antigène CD133 [4]. À partir de ces expériences pionnières, le concept de cellules souches cancéreuses s'est propagé comme une traînée de poudre sans toutefois être toujours validé expérimentalement de façon rigoureuse, ce qui est plus difficile dans les cancers solides que dans les hémopathies, les cellules leucémiques étant facilement accessibles dans le sang ou la moelle osseuse, et la hiérarchie hématopoïétique solidement établie.

\section{Patatras}

Or, un article récent de l'équipe de Quintana [5] remet en cause la « rareté » des cellules de type CSC en démontrant que dans les mélanomes humains, $25 \%$ des cellules cancéreuses sont capables d'induire une tumeur in vivo, alors que des articles antérieurs faisaient état d'une fréquence de 1 sur $10^{6}$ cellules [6]. Cette divergence vient de la simple modification par Quintana du protocole expérimental de greffe in vivo xénogénique sur lequel repose toute démonstration de l'existence de cellules souches cancéreuses humaines. Les modifications introduites par Quintana concernent trois paramètres importants: (1) l'allongement du temps d'observation, de huit semaines conventionnelles à 32 semaines, ce qui permet de détecter 4 fois plus de tumeurs «palpables» in vivo; (2) l'utilisation de souris (NOD/SCID/IL2R $\left.\gamma^{-/-}\right)^{l}$, dont le déficit immunitaire est plus profond que celui des NOD-SCID «standard», notamment par l'absence de cellules natural killer [7]; et (3) l'amélioration de la survie des cellules tumorales greffées par leur co-injection avec du matrigel, contenant notamment de la laminine, substrat naturel présent dans l'environnement tumoral in vivo. Dans ces conditions, des expériences d'injection de nombres décroissants de cellules tumorales aux souris permettent d'évaluer la fréquence des cellules capables de former une tumeur in vivo à une cellule sur quatre lorsque les souris NOD/SCID/IL2R $\gamma^{-/-}$sont utilisées comme receveuses, et moins de 1 cellule sur 50-100000 si l'on utilise des souris NOD/SCID comme c'était fait jusqu'à maintenant. Cette proportion importante de cellules capables de former des tumeurs a été confirmée en transplantant des cellules tumorales uniques (incluses dans du matrigel) à des souris NOD/SCID/IL2R $\gamma^{-/-}$- le gold standard expérimental pour évaluer le caractère «souche»d'une cellule, $12 \%-65 \%$ des cellules formant des tumeurs. Dans ces expériences, pour des raisons de facilité expérimentale, les cellules tumorales testées sont issues de tumeurs développées chez des receveurs xénogéniques primaires (les auteurs ont vérifié que le passage in vivo n'augmentait pas la tumorigénicité), mais les résultats ont été vérifiés avec des cellules directement isolées de tumeurs humaines. Autre divergence avec certaines données récentes, l'impossibilité d'identifier des marqueurs spécifiques de ces cellules capables de produire une tumeur. II est donc possible que, dans les mélanomes, l'observation dans les études antérieures d'une fréquence faible

\footnotetext{
${ }^{1}$ NOD-SCID : non obese diabetic severe combined immunodeficient; ces souris ont été croisées avec des souris dont le gène codant la chaîne $\gamma$ des récepteurs de cytokines (notamment I'IL-2, I'IL-7 et I'IL-15) a été inactivé. Chez ces souris NOD-SCID $\times$ IL2R $\gamma^{-1-}$, la lignée natural killer est absente, comme le sont les lignées lymphoïdes $B$ et $T$, et ce déficit immunitaire plus profond permet une bien meilleure reconstitution hématopoïétique après transplantation de cellules souches hématopoïétiques humaines à ces souris.
} 
de cellules capables de former des tumeurs ait été en partie due à des limitations du système expérimental xénogénique: persistance d'une réponse immunitaire murine, mort des cellules humaines greffées sevrées de leur environnement, et dont les récepteurs de cytokines sont peu sensibles aux ligands murins, empêchant des interactions nécessaires à la survie/prolifération des cellules transplantées. A. Strasser incriminait déjà ces limitations du système xénogénique en 2007 en démontrant dans des lymphomes murins une fréquence très élevée de cellules capables d'établir des tumeurs in vivo chez des hôtes congéniques histocompatibles [8].

\section{De la diversité en toutes choses}

Ces résultats représentent-ils un coup d'état contre le théorème en place? Plutôt une interrogation saine sur la limite des techniques et modèles expérimentaux classiques que nous utilisons pour établir nos concepts.
Les méthodes actuelles d'identification et d'isolement des CSC, dont la formation de «sphères » (mammo-, prostato, colonosphères), le développement de colonies et la détection de marqueurs spécifiques ont leurs limites. En particulier, peu se soucient de l'influence du microenvironnement tumoral, qui est rarement reconstitué dans ces systèmes. Or on connaît maintenant l'importance de l'influence de la signalisation par les cellules stromales entourant la tumeur non seulement sur l'expression des marqueurs antigéniques, mais aussi sur la survie et la croissance des CSC. Ces résultats obtenus dans les mélanomes ne sont peut-être pas généralisables, et d'autres tumeurs cachent sans doute de vraies et rares «cellules souches» cancéreuses, mais ils nous renvoient à la paillasse et confirment l'importance de la diversité et le danger des dogmes... $\diamond$
The shaking oligarchy

of cancer stem cells

\section{RÉFÉRENCES}

1. Clarke MF, Dick JE, Dirks PB, et al. Cancer stem cells: perspectives on current status and future directions: AACR Workshop on cancer stem cells. Cancer Res 2006; $66: 9339-44$.

2. Bonnet $D$, Dick JE. Human acute myeloid leukemia is organized as a hierarchy that originates from a primitive hematopoietic cell. Nat Med 1997 ; $3: 730-7$.

3. Eisterer W, Jiang X, Christ 0 , et al. Different subsets of primary chronic myeloid leukemia stem cells engraft immunodeficient mice and produce a model of the human disease. Leukemia $2005 ; 19$ : 435-41.

4. Oliver TG, Wechsler-Reya RJ. Getting at the root and stem of brain tumors. Neuron $2004 ; 42: 885-8$.

5. Quintana $\varepsilon$, Shackleton M, Sabel MS, et al. Efficient tumour formation by single human melanoma cells. Nature 2008; 456: $593-8$.

6. Schatton T, Murphy GF, Frank Ny, et al. Identification of cells initiating human melanomas. Nature 2008 ; 451 : 345-9.

7. Agliano A, Martin-Padura I, Mancuso P, et al. Human acute leukemia cells injected in NOD/LtSz-scid/IL-2Rgamma null mice generate a faster and more efficient disease compared to other NOD/scid-related strains. Int J Cancer $2008 ; 123: 2222-7$.

8. Kelly PN, Dakic A, Adams JM, et al. Tumor growth need not be driven by rare cancer stem cells. Science 2007; $317: 337$.

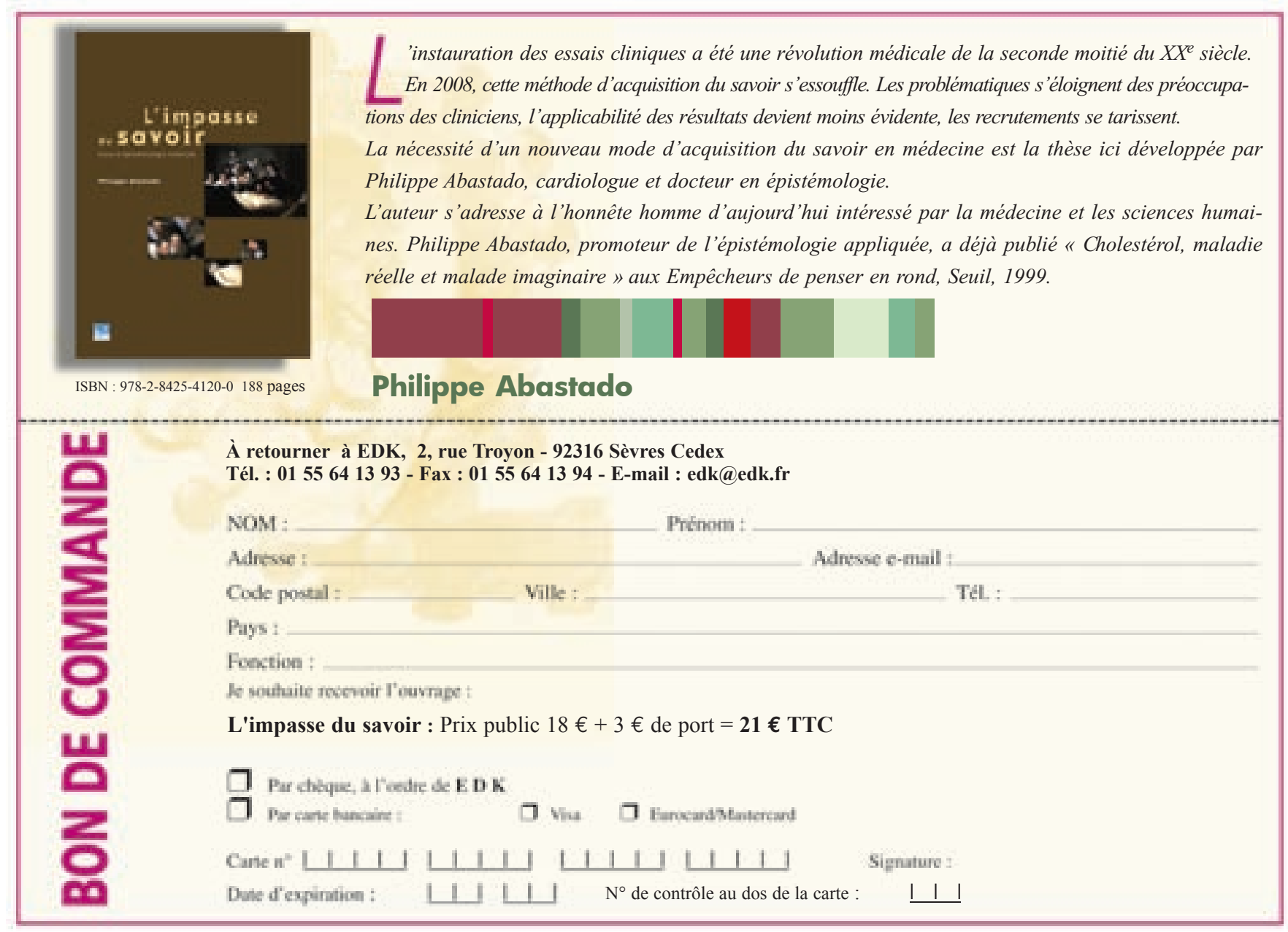

